OPEN

\title{
Gut Permeability and Glucose Absorption Are Affected at Early Stages of Graft Rejection in a Small Bowel Transplant Rat Model
}

Pablo Stringa, PhD, ${ }^{1,2,3}$ David Romanin, ${ }^{1}$ Natalia Lausada, ${ }^{2}$ Rodrigo Papa Gobbi, ${ }^{1}$ Carolina Zanuzzi, ${ }^{4}$ Pedro Martín, ${ }^{1}$ Juan Cruz Abate, ${ }^{2}$ Ana Cabanne, ${ }^{5}$ Nathalie Arnal, ${ }^{6}$ Leandro Vecchio, ${ }^{2}$ Verónica Milesi, ${ }^{1}$ Enrique Portiansky, ${ }^{4}$ Gabriel Gondolesi, ${ }^{3,5}$ and Martin Rumbo ${ }^{1}$

Background. Intestinal transplantation (ITx) faces many challenges due to the complexity of surgery and to the multiple immunological reactions that lead to the necessity of rigorous follow-up for early detection of acute cellular rejection (ACR). Our aim was to determine the kinetics of ACR using an experimental ITx model, with emphasis in the characterization of the process using different approaches, including the use of functional assays of absorptive and barrier function. Methods. ITx in rats conducting serial sampling was performed. Clinical monitoring, graft histology, proinflammatory gene expression, and nitrosative stress determination were performed. Also, glucose absorption, barrier function using ovalbumin translocation, and contractile function were analyzed. Results. The model used reproduced the different stages of ACR. Allogeneic ITx recipients showed signs of rejection from postoperative day (POD) 5, with increasing severity until 12 POD. Histological evaluation showed mild rejection in early sampling and severe rejection at late stages, with alterations in all graft layers. IL-6, CXCL 10, IFNg, and nitrite plasmas levels showed behavior coincident with histopathology. Remarkably, allogeneic grafts showed a marked alteration of glucose absorptive capacity from POD 5 that was sustained until endpoint. Coincidently, barrier function alteration was evidenced by luminal ovalbumin translocation to serum. Contractile function was progressively impaired along ACR. Conclusions. Glucose absorption and barrier function are altered at early stages of ACR when histological alterations or gene expression changes were much subtle. This observation may provide simple evaluation tools that could be eventually translated to the clinics to contribute to early ACR diagnosis.

(Transplantation Direct 2017;3:e220; doi: 10.1097/TXD.0000000000000718. Published online 6 October, 2017.)

ntestinal transplantation (ITx) faces many challenges, due to the complexity of the surgical technique, the multiple immunological reactions that occur during and after engraftment

Received 21 June 2017. Revision requested 22 June 2017.

Accepted 23 June 2017.

${ }^{1}$ Instituto de Estudios Inmunológicos y Fisiopatológicos (IIFP-CONICET-UNLP), La Plata, Argentina.

${ }^{2}$ Laboratorio de Trasplante de Organos y Tejidos (LTO-UNLP), La Plata, Argentina. ${ }^{3}$ Instituto de Medicina Traslacional, Trasplante y Bioingeniería (IMETTYB-CONICET), Buenos Aires, Argentina.

${ }^{4}$ Laboratorio de Imágenes (CONICET-UNLP), La Plata, Argentina.

${ }^{5}$ Instituto de Trasplante Multiorganico, Hospital Universitario Fundacion Favaloro, Buenos Aires, Argentina.

${ }^{6}$ Instituto de Investigaciones Bioquímicas de La Plata (INIBIOLP-CONICET-UNLP), La Plata, Argnetina.

This work was financed by grants from Agencia Nacional de Promoción de la Ciencia y la Tecnología (ANPCYT) to Dr. Gabriel Gondolesi.

P.L.S. is fellow from Argentinean Nacional Research Council (CONICET), L.V. is fellow from Universidad Nacional de La Plata. D.R., G.G., V.M., and M.R. are members of CONICET.

The authors declare no conflicts of interest.

P.S. performed and designed the experiments, surgical procedures, collected and analyzed the data, and in article writing. D.R. performed and designed the experiments, collected and analyzed the data, and participated in article writing. $\mathrm{N}$. and the necessity of rigorous follow-up for early detection of rejection processes. ${ }^{1}$ Rodent models of small bowel transplantation were used to provide evidence regarding intervention strategies, to understand immunological features of ITx as well

\footnotetext{
L., L.V., and J.C.A. performed the surgical procedures. R.P.G. performed the principal component analysis of gene expression and ELISA determinations. C.Z. and E.P. performed Tunel technique and collagen determinations, collected and analyzed the data. N.A. performed nitrosative stress determination, collected and analyzed the data. V.M. and P.M. performed graft contractile evaluation, collected and analyzed the data. A.C. performed histological analysis of transplanted intestines. G.G. and M.R. designed the experiments, analyzed the data and participated in article writing.

Correspondence: Pablo Luis Stringa, PhD, Facultad de Ciencias Exactas, Instituto de Estudios Inmunológicos y Fisiopatológicos, Calle 47 y 115, La Plata CP:1900, Argentina. (pstringa7@gmail.com;pablo_stringa@hotmail.com).

Supplemental digital content (SDC) is available for this article. Direct URL citations appear in the printed text, and links to the digital files are provided in the HTML text of this article on the journal's Web site (www.transplantationdirect.com).

Copyright () 2017 The Author(s). Transplantation Direct. Published by Wolters Kluwer Health, Inc. This is an open-access article distributed under the terms of the Creative Commons Attribution-Non Commercial-No Derivatives License 4.0 (CCBY-NC-ND), where it is permissible to download and share the work provided it is properly cited. The work cannot be changed in any way or used commercially without permission from the journal.
}

ISSN: 2373-8731

DOI: $10.1097 / T X D .0000000000000718$ 
as to improve our knowledge on ITx biology. ${ }^{2}$ By far, rat ITx models has been the most extensively used mainly as consequence of technical aspects and the availability of different strains. ${ }^{3}$ Since the standardization of procedures in the last decade of the XXth. century, orthotopic ITx and heterotopic ITx (HITx) variants of ITx are used, each of them allowing the evaluation of different aspects of the process. ${ }^{4,5}$ Despite HITx models do not reproduce the physiological anatomy of an orthotopic procedure, they are commonly used to study immunological reactions because they have the advantages of being technically easy, having higher survival rate, and allowing serial sampling of the graft. The HITx has been used to establish the proof of concept of different immune modulation strategies to avoid rejection, as well as to study immunological reactions involved in graft rejection. ${ }^{6-10}$

We here report the use of a HITx model to study the impact of the initial stages of acute cellular rejection (ACR) on graft functional features, such as absorptive capacity, barrier function, and motility. We analyzed the kinetics of these variations in the context of the characterization of clinical and molecular changes associated with the ACR process.

\section{MATERIALS AND METHODS}

\section{Animal Use and Care}

Adult Wistar and Sprague-Dawley male rats (average weight, $220 \pm 15 \mathrm{~g}$ ) were housed in a climate-controlled room on a 12-hour light-dark cycle, fed with standard laboratory rats chow, and allowed water ad libitum. Wistar rats were provided by the Facultad de Ciencias Veterinarias of the Universidad Nacional de La Plata (Argentina) animal facility and SpragueDawley animals were provided by the Facultad de Ciencias Veterinarias of the Universidad de Buenos Aires (Argentina). All animals received human care, and the study protocols complied with local guidelines for vertebrate animal welfare as well as with USPHS and/or European Union policy on this matter.

\section{Surgical Procedure and Experimental Protocol}

HITx was performed under general anesthesia (isofluorane). The surgical technique was like those previously described ${ }^{3}$ (Materials and Methods, SDC, http://links.lww.com/ TXD/A52).

Animals were divided into following groups per strain combination: (1) isogenic (ISO) HITx group (Wistar as donor and recipient) and (2) allogeneic (ALLO) HITx group (SpragueDawley as donor, Wistar as recipient).

Intestinal graft was sampled from recipient distal stoma at 30 minutes, 3, 5, and 7 postoperative days (POD) after HITx and at the end point of the experimental protocol (10-12 POD). Also, blood samples from tail vein were taken at 5, 7 and 10 to 12 POD.

\section{Recipient and Graft Clinical Monitoring}

Recipient clinical monitoring after HITx in search of pain and/or discomfort signs was daily performed and quantified. Ocular secretion, hair appearance, posture, attitude, and feces production was considered as postsurgical discomfort signs. Each recipient received a general score resulting from adding each evaluated parameter (Figure S1, SDC, http://links.lww. com/TXD/A52). Also, recipient body weight was daily evaluated. Graft monitoring by abdominal palpation was performed every 24 hours. Graft tightening was considered as a clinical indicator of ACR as previously described.

\section{Functional Evaluation of the Transplanted Intestine}

To evaluate graft absorptive and barrier function, a solution containing glucose $(2 \mathrm{~g} / \mathrm{kg})$ and ovalbumin (OVA) $(150 \mu \mathrm{g} /$ dose) diluted in $1.5-\mathrm{mL}$ saline was administered by proximal ostomy 5 and 10 days after ITX. Glycemia was measured using an Accu-Chek blood glucose meter (Roche) just before and 15, 30, 60, 90, 120, and 180 minutes after glucose administration. Serum levels of OVA were measured by competitive ELISA 30 and 90 minutes after administration as previously described. ${ }^{11}$ For both measurements, blood samples were obtained from the receptor tail vein. Also, graft contractile function by isometric graft force measurements was analyzed at 4 and 10 POD (Materials and Methods, SDC, http://links.lww.com/TXD/A52).

\section{Histology and Immunohistochemistry}

Histological analysis of transplanted intestines was performed on hematoxylin-eosin-stained 5 - $\mu \mathrm{m}$ tissue sections. All samples were analyzed by experienced pathologist in a blinded way. ACR was determined using 2 different scores previously used in ITx animal models. ${ }^{12,13}$ Also, ischemia-reperfusion damage using Park score were determined. ${ }^{14}$ Besides, a descriptive evaluation of each intestinal graft layer in search for histological alterations was performed.

An immunohistochemical study to evaluate apoptosis was performed by Tunel technique using the In-Situ Cell Death TMR (Roche) following manufacturer's instructions. Counting of apoptotic cells was performed considering the number of apoptotic cells in 10 fields per sample using a confocal microscope (Olympus FV1000). Apoptotic cells were initially counted in the whole wall of the graft. Subsequently, the total number of cells observed in each sample was classified per their location in the transplanted intestine (lamina propria, crypts or intestinal epithelium compartment).

\section{Collagen Determination}

Collagen evaluation was done in sections stained with the Picrosirius red technique and viewed with polarized light (Montes, 1996). For this purpose, sections were deparaffinized, hydrated through graded ethanol and stained for 1 hour in a $0.1 \%$ solution of Sirius Red (Direct Red 80; Aldrich, Milwaukee, WI) dissolved in aqueous saturated picric acid. Sections were then rapidly washed in running tap water and counterstained with Harris hematoxylin (Montes 1996). A conventional optical microscope (BX53 Olympus microscope, Japan) with a strong light source (halogen lamp), an analyzer (U-ANT Olympus, Japan) and a polarizer (U-POT Olympus, Japan) were used to study the birefringence of the stained collagen. ${ }^{15}$

\section{Laser Microdissection}

Laser microdissection was performed as previously described. ${ }^{16}$ Briefly, intestinal segments were fixed overnight in zinc-sucrose fixation. Frozen intestinal sections of $15 \mu \mathrm{m}$ were obtained with Shandon Cryotome from Thermo Scientific (Waltham, MA) at $-20^{\circ} \mathrm{C}$ and placed on membrane Slides PEN-Membrane 2,0 $\mu \mathrm{m}$ (Leica, Germany). Tissue sections were fixed in $70 \%$ ethanol for 1 minute stained with haematoxylin for 30 seconds and putted in 100\% ethanol for 1 minute. Cells fractions from epithelium, muscular layer, and serosa were obtained with microscope LMD6000 from Leica and collected in lysis buffer for RNA isolation. As average 30 microdissected pieces were collected in a single tube. 
TABLE 1.

Rat primers used to evaluate gene expressions

\begin{tabular}{lc}
\hline Primer & Sequence \\
\hline qRat IL22 fwd & TGG TGC CT TCC TGA CCA A \\
qRat IL22 rev & GT CTG GTC ATC ACC GCT GAT \\
qRat IFNg fwd & TAC ACG CCG CGT CTT GGT \\
qRat IFNg rev & GAG TGT GCC TTG GCA GTA ACA G \\
qRat CXCL1 fwd & TGGATGCGTTCATCGATGGTCGT \\
qRat CXCL1 rev & GCACAGTGGTGACACCTAATGGT \\
qRat ID0 fwd & CAG CAG ATC CT CAC CAA CG \\
qRat ID0 rev & ATC CAG ACA CCT TाT TCC ACG \\
qRat CXCL10 fwd & CTGCACCTGCATCGACTCC \\
qRat CXCL10 rev & TCTTTGGCTCACCGCTTC \\
qRat IL13 fwd & GCA ACA TCA CAC AAG ACC AGA AG \\
qRat IL13 rev & TGT CAG GTC CAC GCT CCA T \\
qRat actB fwd & 9Rat actB rev CTGACCCATACCCACCATCAC \\
\hline
\end{tabular}

RNA Extraction, Reverse Transcription, and Quantitative Polymerase Chain Reaction

Total RNA extraction from microdissection or whole intestinal graft was performed using the NucleoSpin RNA II kit (GE Healthcare, Aurora, OH). Reverse transcription was performed using random primers and MMLV-Reverse transcriptase (Invitrogen, Carlsbad, CA). Real-time polymerase chain reaction was performed following manufacturer's protocol using the iCycler thermal cycler (BioRad, Philadelphia, PA). Primers for rat IL6, IL22, IFNg, CXCL1, IDO, CXCL10, IL13, and Actin-b were designed by us or adapted from literature, and their sequences are described in Table 1. Relative difference calculation using the $\Delta \Delta \mathrm{Ct}$ method was previously described. ${ }^{16,17}$

\section{Nitrosative Stress Determination}

Nitrate and nitrite concentration was measured using the method of Griess on samples previous reduced with vanadium hydrochloride according to Miranda et al. ${ }^{18}$ Quantification was performed after calibration with standard solutions of sodium nitrate from Merck Co. (Darmstadt, Germany). Protein carbonyls (PCs) were determined by the method of Reznick et al. ${ }^{19}$ The concentrations of PCs were calculated from a calibration curve prepared with a stock solution of sodium pyruvate (Sigma Chem. Co.).

\section{Data Analysis}

Continuous variables were analyzed using 1-way analysis of variance, followed by Dunnett or Tukey posttest. Student unpaired $t$ test was used to determine the significance of differences between 2 groups. The Kaplan-Meier method was used for actuarial graft survival with comparison using the Log-rank test. Multivariate analysis of gene expression in early and late ISO and ALLO intestinal graft was performed. Statistical analyses were performed using GraphPad software version 5.00 (San Diego, CA). $P$ values less than 0.05 were considered statistically significant.

\section{RESULTS}

\section{Postoperative Clinical Monitoring}

ALLO and ISO HITx recipients showed similar clinical findings associated to the surgical procedure during the first
96 hours post-HITx.ISO recipients reached a normal clinical appearance from the fifth POD, but ALLO recipients worsened their clinical status with increased ocular/nose secretion and piloerection as the most characteristic clinical affections. Statistically significant differences were found between ISO versus ALLO groups $(P<0.05$ at 5 POD and $P<0.01$ from sixth to 12 th days after ITx) (Figure 1A).

Regarding body weight evaluation after HITx, animals from both groups lost weight during the first 5 postsurgical days (media of 12.7 and $21.1 \%$ in ISO and ALLO groups). From sixth to 12th POD, ALLO recipients showed significant weight loss in comparison with ISO group, which began to gain weight and reached their original body weight 10 to 12 POD. Statistically significant differences were found between ISO versus ALLO groups at 10 to $12 \mathrm{POD}(P<0.01)$ (Figure 1b).

Clinical graft rejection diagnosed by tightening of transplanted intestine was performed. Graft tightening at 7 (3/7), 9 (1/4),
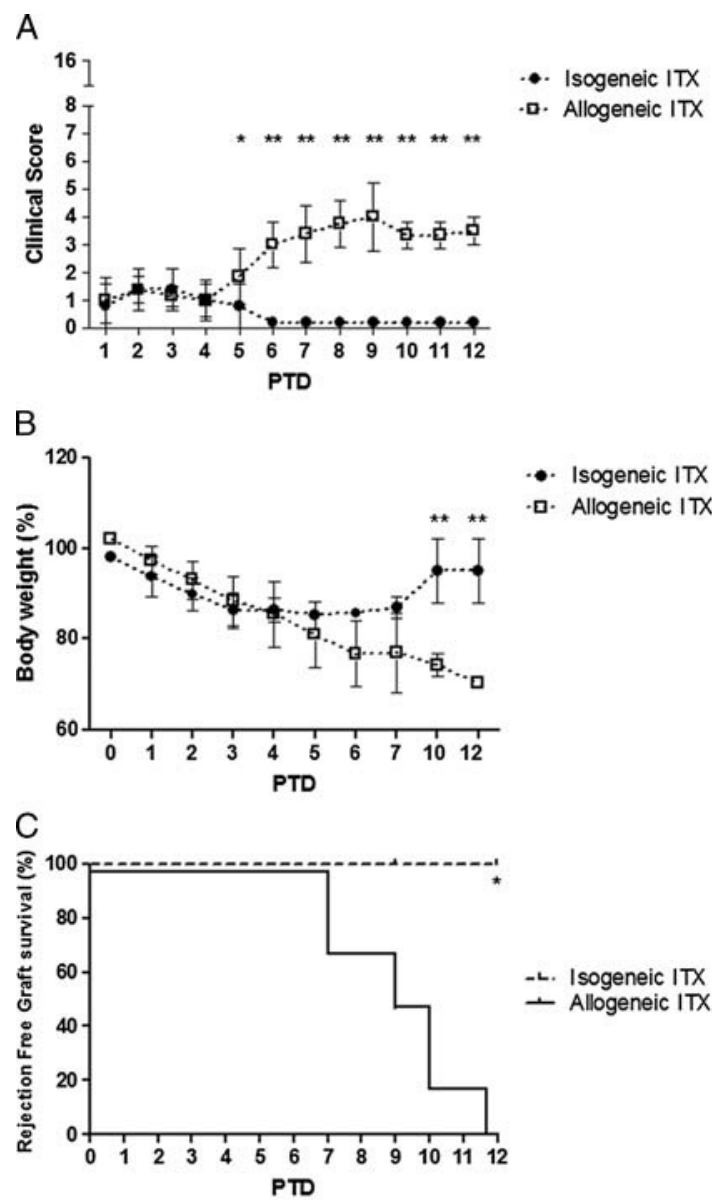

\begin{tabular}{|c|c|c|c|c|c|}
\hline GROUP & $0-6$ & 7 & 9 & 10 & 12 \\
\hline ISO & $0 / 10$ & $0 / 10$ & $0 / 10$ & $0 / 10$ & $0 / 10$ \\
\hline ALLO & $0 / 10$ & $3 / 10$ & $2 / 7$ & $3 / 5$ & $2 / 2$ \\
\hline
\end{tabular}

FIGURE 1. Clinical status of recipients and transplanted intestines are altered during ACR process. A, quantitative clinical score was determined for ALLO and ISO ITx recipients. ${ }^{* \star} P<0.01$ (B) Worsening of ALLO group is also reflected in weight loss compared with ISO group at 10 to 12 days after transplantation. ${ }^{* *} P<0.01$ (C) Graft tightening (used as clinical rejection indicator) was evident in ALLO group in contrast with ISO ITx recipients. ${ }^{\star \star \star \star} P<0.001$ ( $\mathrm{N}=10$ in each group for each evaluation). 
$10(1 / 3)$, and 12 (2/2) POD were diagnosed in ALLO recipients. ISO group showed no palpable abnormalities. Recipients survived healthy up to 6 months after HITx without grafts abnormalities. Significant differences in graft survival between groups were observed $(P<0.001)$ (Figure 1C).

\section{Graft Functional Evaluation}

To evaluate changes in functional graft parameters that are susceptible to be evaluated in the HITx, a bolus of OVA and glucose was administered by the proximal ostomy of the graft and both levels were evaluated at subsequent time points. Nontransplanted controls were included.

Remarkably, glucose absorption was evident in ISO groups at 5 and 10 to 12 POD. Glucose levels rise upon administration reaching maximum levels at 60 to 90 minutes postadministration. Similar curves were obtained from non-ITx controls, as well as in ISO group when glucose was administered by intraperitoneal route, indicating full absorption of the administered glucose at the time observed (Figure 2A). Remarkably, in ALLO groups, at 5 and 10 to 12 POD, glucose levels did not change from the initial value, indicating lack of absorptive capacity, even from the initial stages of rejection. Interestingly, glucose levels were the lowest in ALLO group 10 to 12 POD.

The analysis of plasma levels of OVA at different time postenteral administration allowed the evaluation of intestinal barrier function. OVA was detected at very low levels at different times postadministration in nontransplant controls as well as in ISO groups (Figure 2b), whereas in the ALLO groups at 5 post-TX and 10 to 12 POD, OVA passed through the intestine into the systemic circulation with a plasma mean value that was higher than $1 \mu \mathrm{g} / \mathrm{mL}$ in all cases, 90 minutes after administration. This result indicates that even in early stages of ACR, there is an impairment of barrier function that is evidenced by passage of nondegraded proteins from intestinal lumen to blood.

Contractile capacity was also evaluated to determine the impact of ACR in graft motility. The maximal contractile strength was diminished in the presence of ACR, being a trend at 5 POD days toward 10 to 12 days after surgical procedure, with a statistical significance between ALLO and ISO groups at 10 to $12 \mathrm{POD}$ (Figure 2C).

\section{Intestinal Graft Histological Evaluation}

Samples obtained at 30 minutes postreperfusion showed the typical ischemia-reperfusion injury lesions, with a median Park score of 3 in both groups. Intestinal mucosa recover was observed in ALLO and ISO groups by POD number 3. At this time of evaluation, samples showed a normal architecture and no histological alterations were identified (Figure S2, SDC, http://links.lww.com/TXD/A52).

Graft lesions compatible with ACR were observed since 5 POD in ALLO group. Overall similar results were observed using the scores described by Wu et $\mathrm{al}^{12}$ and He et al. ${ }^{13}$ The former will be used to describe our findings. Mild rejection was the most characteristic grade of graft rejection. The remaining samples showed indeterminate ACR and no signs of ACR. On the other hand, severe ACR was diagnosed in all ALLO samples taken at 10 to 12 POD (Figure 3A).

A descriptive evaluation of hematoxylin-eosin staining intestinal allografts was performed. Samples showed a wellpreserved architecture at 5 to 7 POD. Also, confluent and loose apoptotic cells in the intestinal epithelium and perivascular infiltrate in mesenteric vessels and muscular and serosa layers were evident (Figure 3B).

Lesions along the transplanted intestines were seen heterogeneously at the late phase of ACR. Significant cellular infiltrate, epithelial damage, absence of enterocytes in some areas of the graft villi, ulcerated areas and necrosis zones, and increase of apoptotic cells in the entire graft were observed (Figure 3B).

The muscular layer showed inflammatory cell infiltrate and increased intercellular edema. Histological changes at the serosa layer were like those reported at 5 to 7 POD, with

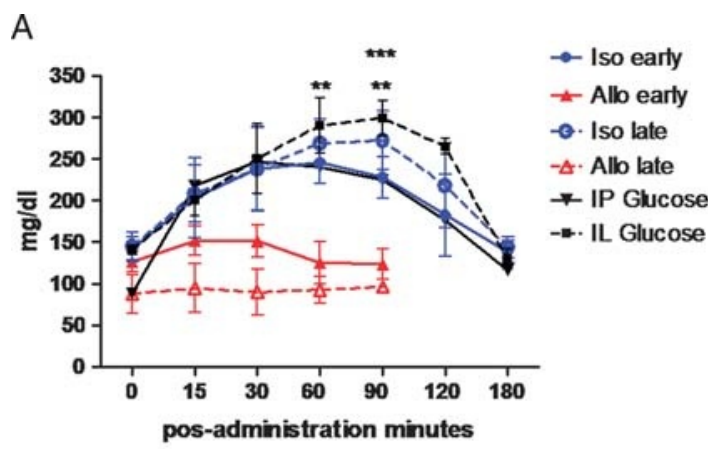

B

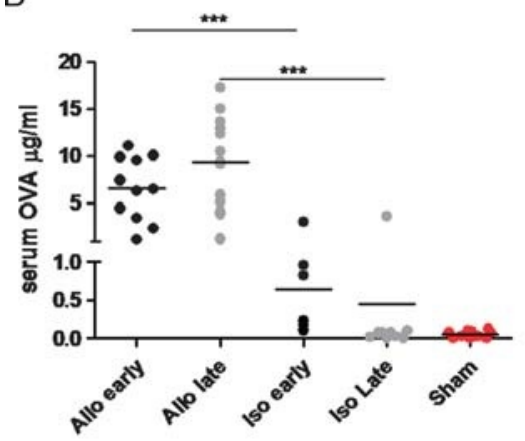

C

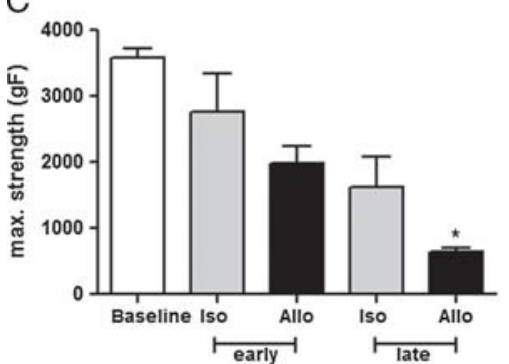

FIGURE 2. Functional features of ALLO intestinal grafts are altered at early stages of ACR. A, Glucose absorptive capacity was evaluated by glucose intraluminal administration and evaluation of glucose blood levels in both early (5 POD) and late (10-12 POD) evaluation times. Significant differences were observed between ALLO versus ISO and control groups (intraperitoneal and intestinal intraluminal administration in nontransplanted animals) 60 and 90 minutes after glucose administration. ${ }^{\star \star} P<0.01$. Mean and SD values of 6 different independent TX procedures are depicted. B, barrier function evaluation performed by plasma OVA determination by ELISA upon administration of OVA through graft proximal ostomy. Significant differences were observed in ALLO vs ISO and Sham groups $\left({ }^{\star \star \star} P<0.005\right)$. Mean and SD values of 6 different independent TX procedures are depicted. C, Maximum contractile strength evaluated by mecanotransduction. Significant differences were observed at late stages of ACR between ALLO vs ISO and control groups. ${ }^{*} P<0.05$. Mean and SD values of 6 different independent TX procedures are depicted. 
A

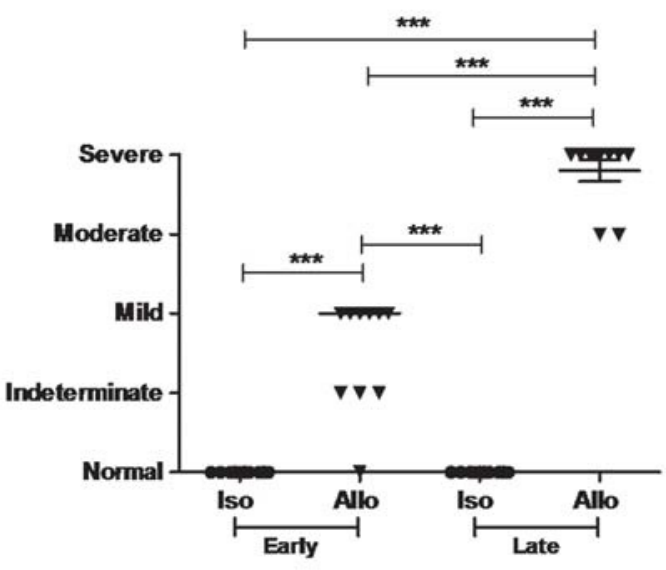

B
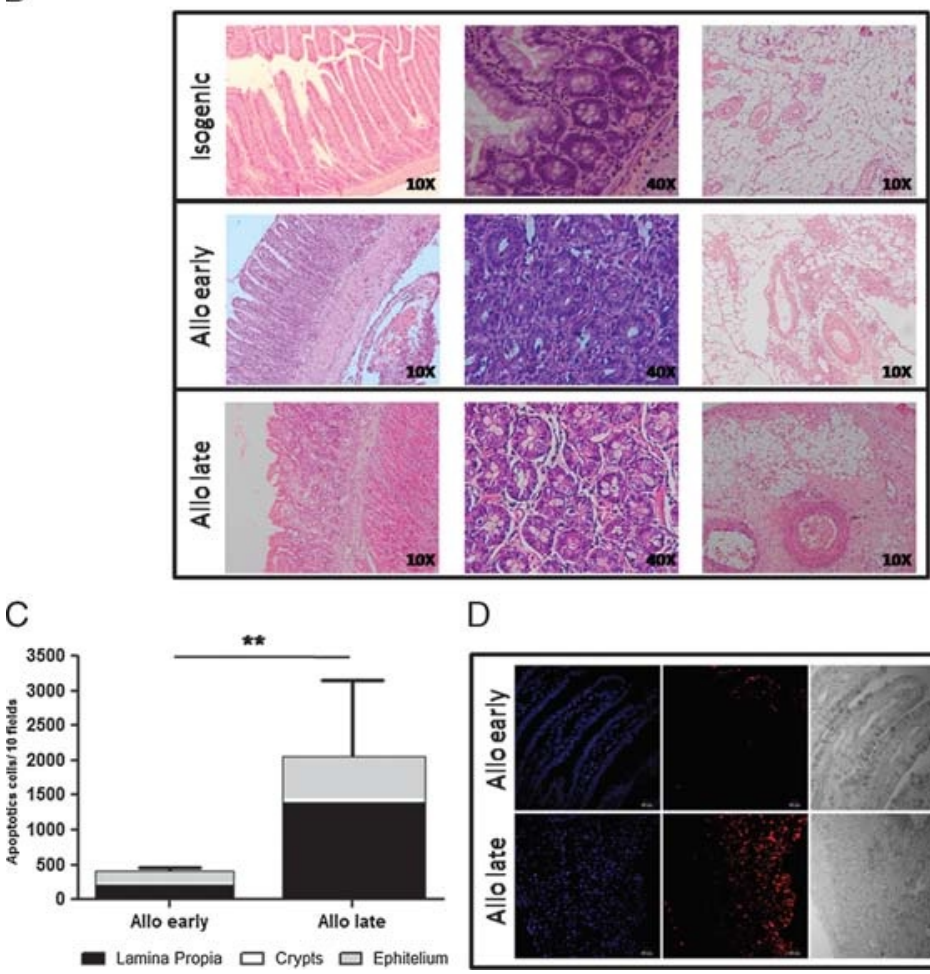

D

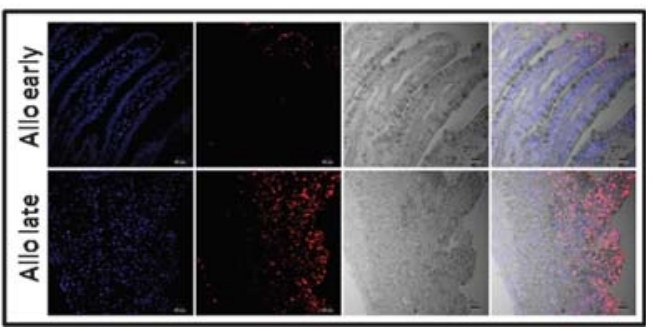

FIGURE 3. Histopathology analysis show evidences of the progression of the ACR process with increasing postoperative time. A, Histologic diagnosis of ACR and morphologic characteristics in ALLO and ISO groups after ITX (N = 10 in each group for each evaluation time). ALLO group showed histopathological sign of ACR from the fifth day after transplantation in contrast to ISO group. ${ }^{\star \star \star} P<0.005$. B. Microscopic graft appearance in H/E stained intestinal samples of ISO and ALLO ITx groups. C, Apoptotic cell quantification and location in ALLO group evidence an increase in the number of apoptotic cells in late stages of ACR. ${ }^{\star \star} P<0.01$. D, Representative images of early and late stages of ACR in the ALLO group (20× magnification) by Tunel assay.

the addition of histological signs compatible with fibrosis (Figure 3B).

Apoptotic cell evaluation by Tunel technique showed a progressive increase in allografts from 5 (average of 679.2) to 12 POD (average of 2021) apoptotic cells in 10 analyzed per sample fields (Figure 3C). Regarding the location of apoptotic cells, most of them were observed in the lamina propria $(56.3 \%$ and $66.6 \%$ at $5 \mathrm{POD}$ and 10 to $12 \mathrm{POD}$, respectively) and intestinal epithelium (38\% and $30 \%$ at 5 POD and 10-12 POD, respectively) in all evaluated sampling times. Apoptotic cells were also observed in crypts of allografts at different time points (5.62 and 3.33 at 5 POD and 10-12 POD, respectively) (Figures 3C and D).

\section{Gene Expression Analysis}

Expression levels of different immune-related genes were evaluated in full-width graft wall biopsies at different postITx times. At day 5, some markers were consistently increased in ALLO group when compared with ISO controls (Figure 4), such as CXCL10 that showed a $120 \pm 80$-fold increase compared with nontransplanted tissue. Furthermore, IFNg and IDO, which are known to be dependent on IFNg stimulation, showed a trend to be increased at these timepoints. Remarkably, other inflammation-related genes, such as CXCL1 and IL6 showed consistent increase in ALLO groups at 10 to 12 POD, when severe rejection process was established (Figure 4A). Interestingly, when a principal component analysis of the 
A
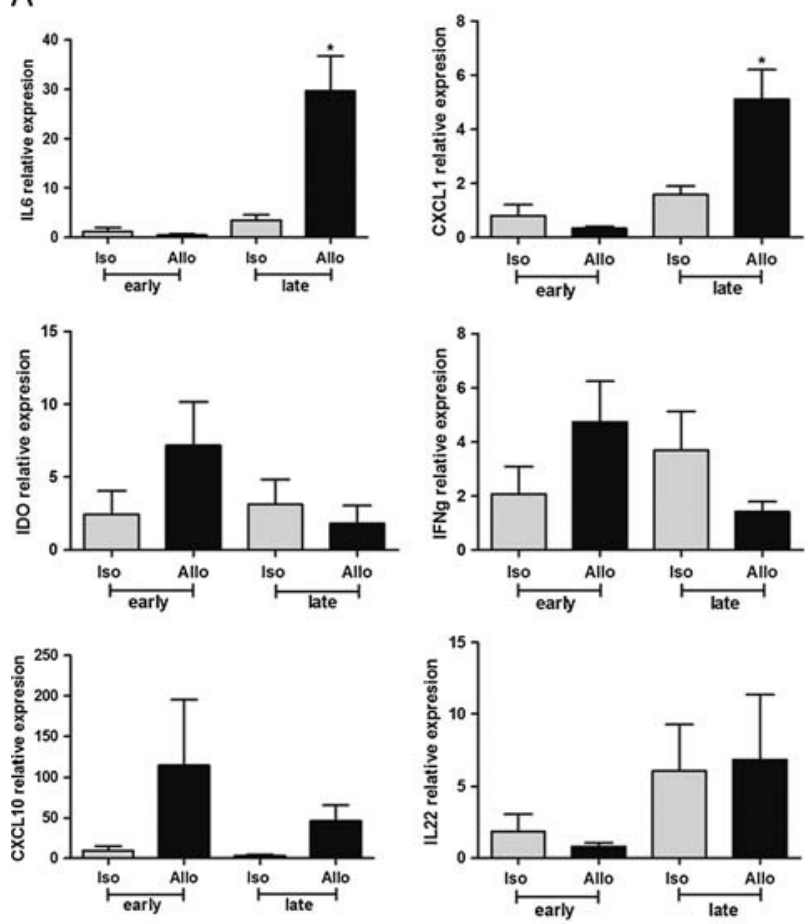

B

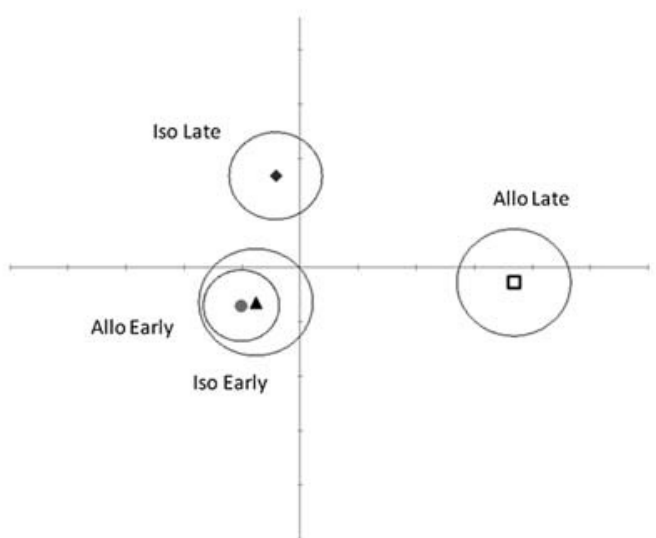

FIGURE 4. Proinflammatory gene expression analysis showed major differences at later stages of ACR process. A, Relative mRNA intestinal proinflammatory gene expression at 5 (early) and 10 to 12 (late) days after ITx in whole intestinal samples in ISO and ALLO groups ( $N=6$ in each group). Significant differences in terms of IL-6 and CXCL-1 expression were observed between later stages of ACR in ALLO group vs ISO group. ${ }^{*} P<0.05$. B, Principal component analysis of gene expression profile in ISO and ALLO ITx groups at different times after ITX. The major difference is observed in the ALLO group during the later stages of ACR.

overall gene expression markers was performed, ALLO group at 10 to 12 POD clearly separated from the other conditions (ALLO group at 5 POD and ISO group at both early and late time points), which were group together (Figure 4b), indicating that major changes in gene expression of the immune related genes evaluated was altered mainly in subsequent stages of ACR.

We wanted to determine if there were differences at the gene expression level among different graft tissue layers during ACR. To this aim, we recovered serosa layer, muscular layer, and epithelial layer using laser dissection microscopy (Figure S3, SDC, http://links.lww.com/TXD/A52) and measured gene expression by quantitative polymerase chain reaction. Overall results from different layers were coincident with whole tissue biopsies (Figure 5), where higher levels of IL6 and CXCL1 were observed in ALLO groups at 10 to 12 POD. Interestingly, IL22 expression was only measurable in epithelial layer samples in ALLO groups at 10 to 12 POD. Serosa layer showed some of the highest relative increases in proinflammatory gene expression also in ALLO groups at 10 to 12 POD (Figure 5).

\section{Collagen Determination}

To characterize the kinetics of extracellular matrix turnover that takes place during the inflammatory processes, collagen type I and type III were determined by the Picrosirius staining technique. We observed that the type I/type III ratio increased in ALLO group at days 10 to 12 post-TX, indicating extensive remodelling (Figure 6).
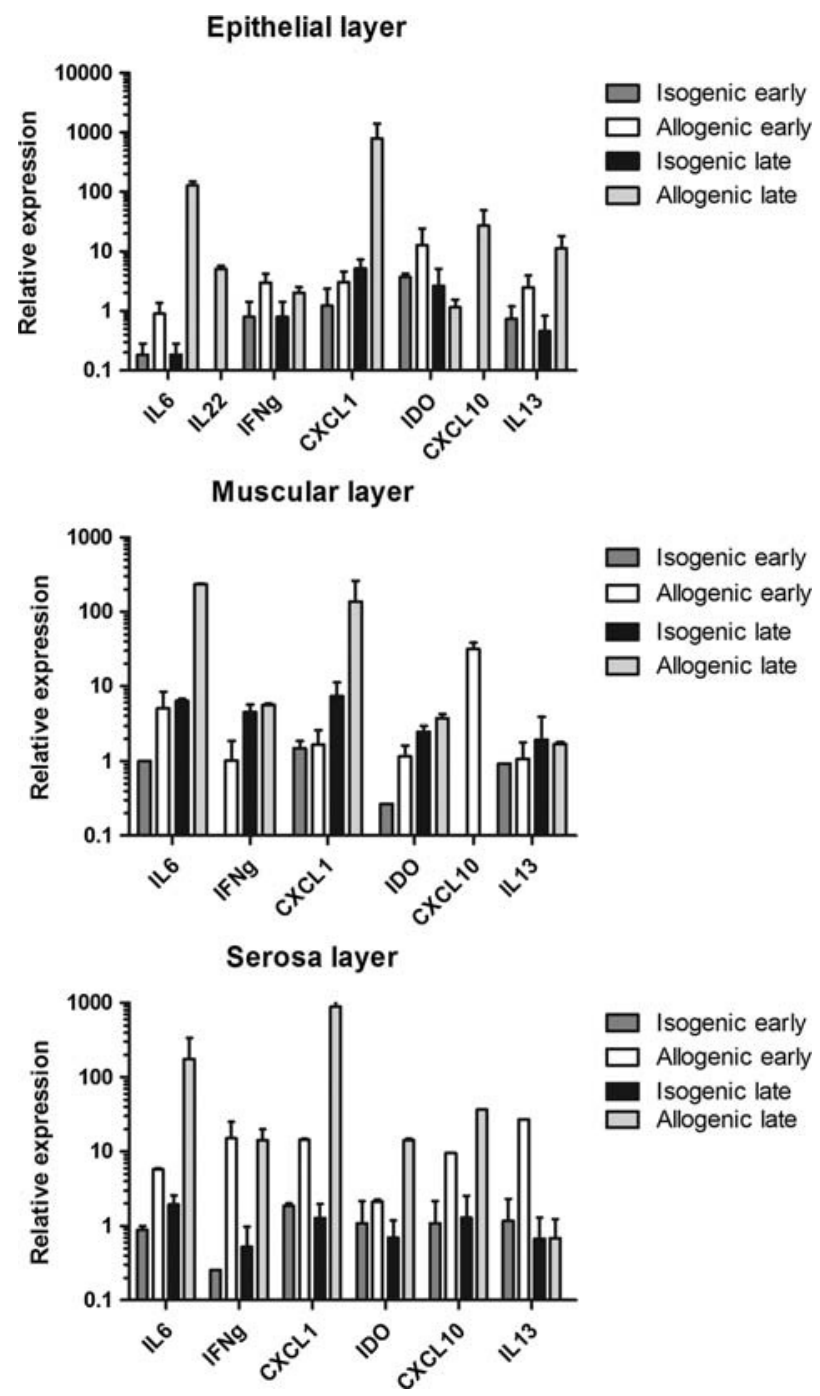

FIGURE 5. Proinflammatory gene expression was increased in different graft tissue layers upon ACR. Epithelial, muscular and serosa layers were obtained by laser microdissection and gene expression in each sample was determined by real-time quantitative polymerase chain reaction ( $\mathrm{N}=5$ for each layer and group). Relative mRNA levels of IL22, IFNg, CXCL1, IDO, CXCL10, IL13 were evaluated. 


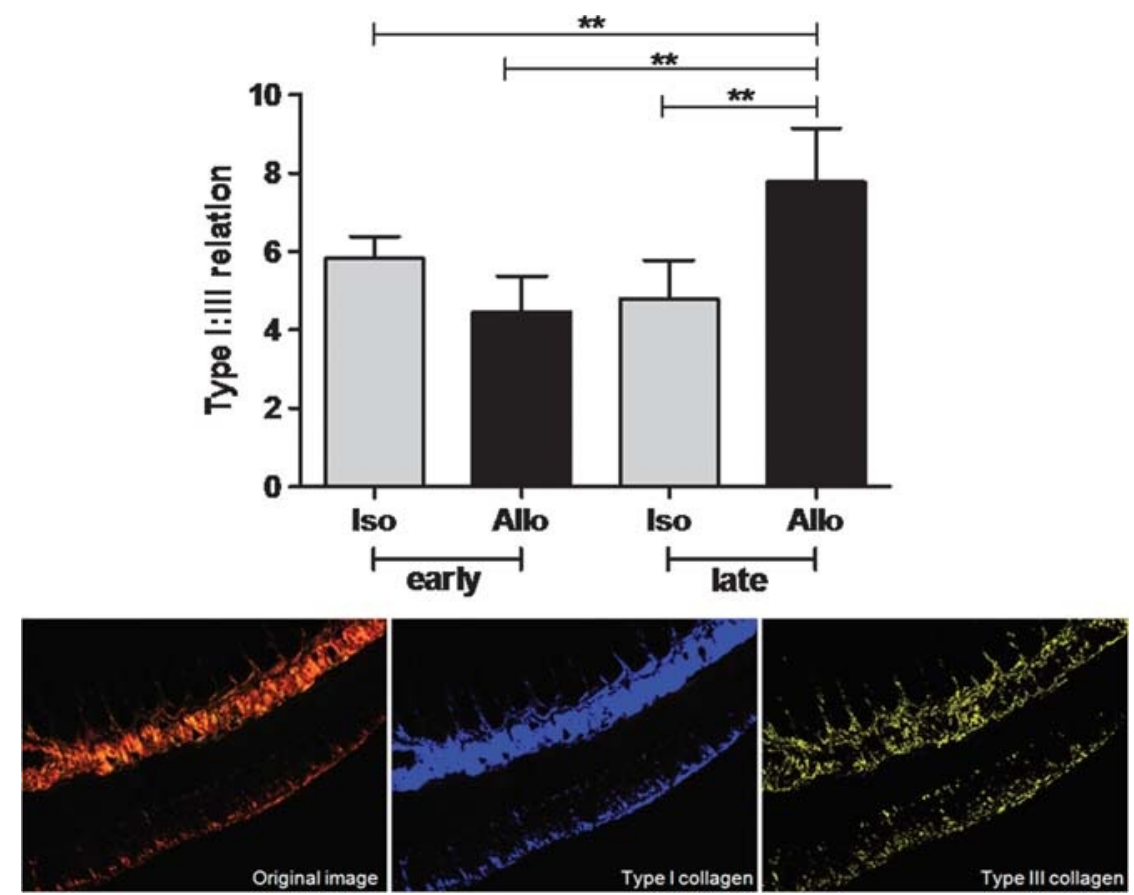

FIGURE 6. Collagen type I/III increase considerably in ALLO group after 10 to 12 days after ITX compared with ALLO ( $N=6$ ) early and early and late ISO groups $(\mathrm{N}=6)$. ${ }^{\star \star} P<0.01$. Also, representative microscopic images of different collagen types are shown.

\section{Plasma Nitrites Levels Increased From the Early \\ Stages of Rejection Process}

To follow the kinetics of putative plasmatic biomarkers of rejection, nitrites and protein carbonyls were evaluated at different time points. Nitrites plasma levels considerably increased in ALLO recipients. The highest levels of nitrites were observed at the early ACR phase, with an average of $568.1 \pm 185.4(P<0.05$ vs baseline $)$. During the late phase of ACR, elevated levels of plasma nitrites were also observed $(558.7 \pm 200.0)$, being statistically significant compared to baseline $(69.5 \pm 22.2 \mathrm{mU} / \mathrm{mL})$ as well as to ISO group. This group showed similar values to baseline in both sampling times, with an average of $118.0 \pm 54.6 \mathrm{mU} / \mathrm{mL}$ and $65.2 \pm 15.0 \mathrm{mU} / \mathrm{mL}$ in the early and late measurements respectively (Figure 7A).

A significant increase in plasma levels of protein carbonyls was only observed in the late phase of the ACR process. Although ALLO group showed an average of $0.0057 \mathrm{mmol} / \mu \mathrm{g}$, the average baseline was $0.0032(P<0.05)$ (Figure $7 \mathrm{~b})$.

\section{DISCUSSION}

We performed a HITx model using a previously reported strain combination. The model using an outbreed strain reproduced the major histopathological findings of clinical rejection, allowing to see in the animal the dynamic process observed in the clinical setting. ${ }^{20}$ Although all results shown were obtained using Sprague Dawley donors into Wistar recipients, the reverse combination produced similar results at the histopathological levels (previously reported). The use of the heterotopic technique without immunosuppression lead to an overt ACR that progress to severe exfoliative rejection in 10 to 14 days, which is similar to other reports using different strain combinations ${ }^{21-23}$ Interestingly, we observed immunological activity with infiltration (Figure 3 ) and collagen turnover (Figure 6) in different tissue layers, mucosa, muscular,

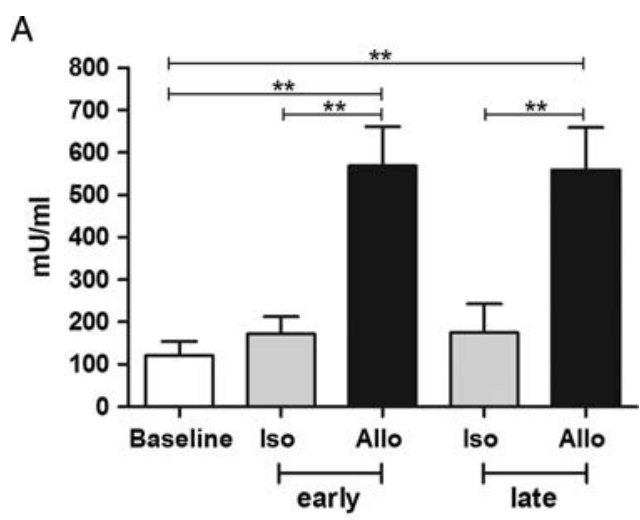

B

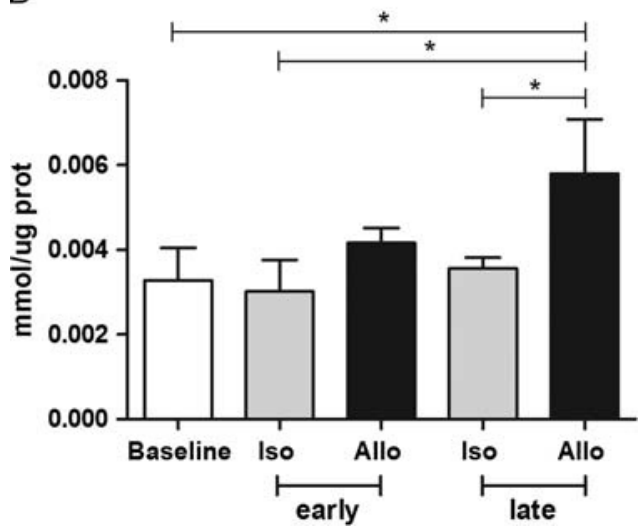

FIGURE 7. A, Nitrites plasma levels increase from initial stages to late phases of ACR process. Significant differences were observed between ALLO $(\mathrm{N}=6)$ vs ISO $(\mathrm{N}=6)$ and basal nitrites levels. ${ }^{\star \star} P<0.01 . \mathrm{B}$, Plasma levels of protein carbonyls only increase significantly in later phases of ACR in ALLO group. ${ }^{*} P<0.05$. 
and serosa, indicating that active allorecognition and rejection affects all tissue layers. During the clinical monitoring, the use of mucosal biopsies has provided extensive information on the changes affecting graft mucosa, during rejection episodes, being the gold standard method for patient follow-up, because deeper layers cannot be biopsied due to methodological limitations. ${ }^{24}$ However, the evidence that ACR compromises other tissue layers, not only the mucosa, could be proved in these model, were the histology (Figure 3) as well as in the gene expression tests (Figure 5), provide information on these regard and might suggest, in the future, new possible molecules to be used as early rejection biomarkers, field in which epithelial-related biomarker were dominant, such as citrulline or fatty acid binding protein. ${ }^{25,26}$

Our results also demonstrate the early activation of IFNg axis during ACR process, because at early time points ( 5 days post-ITx), there is an increase of expression of IFNg-related genes, such as CXCL10 and IDO. Those results are coincident with the rise in circulatory levels of nitrites (Figure 7), because of M1 macrophage activation and NO production, which is also associated to IFNg axis. Their role in the pathophysiology of ACR has also been reported. ${ }^{27}$ These results validate previous reports, indicating that IFNg is a crucial driver of ACR in ITx. ${ }^{28,29}$

One interesting finding of our experiments is the early loss of graft functional capacity as ACR progress. We used very simple techniques with tracers that could be easily translated to a clinical setup, such as glucose testing and OVA evaluation by ELISA. It has been previously reported that monosaccharide absorption capacity is impaired by ACR in rodent ITx models by 14 POD. ${ }^{30}$ In our set of experiments, we could determine that loss of absorptive capacity for glucose is an early feature of ACR, because when only mild alterations were detected by histopathological analysis, the glucose absorption was completely blunted to levels similar to observed at severe exfoliative rejection (Figure 2). This could be due to the fact that glucose is normally absorbed through the transcellular route after an active process that requires enterocyte energy fitness. Although not fully elucidated at the cellular and molecular levels, there is consensus that alloactivation of CD4 and CD8 lymphocytes is the main driver of graft rejection, with enterocytes being one of the main targets. ${ }^{31}$ Increase in crypt enterocyte apoptosis is 1 characteristic feature of small bowel rejection..$^{32-34}$ Glucose absorption takes place in villous epithelium, consequently, our observation may indicate that villous enterocytes are also affected by initial rejection events. Lymphocytes suffer from several important metabolic changes upon activation, with an increase in glycolysis being one of the most constant features. ${ }^{35}$ Consequently, increased local glucose consumption by activated lymphocytes in the rejecting graft may also contribute to the lack of increment in glucose levels in peripheral circulation.

On the other hand, the use of enteral OVA administration allowed determining the loss of barrier function. In physiological situations, ingested proteins are almost completely degraded along the gastrointestinal tract, and only a minimal fraction can reach the systemic compartment as nondegraded protein by passage through paracellular route. ${ }^{36-38} \mathrm{We}$ confirmed that either in nontransplanted animals, the administration of enteral OVA does not result in presence of immunoreactive OVA in circulation at either 30 or 90 minutes postadministration. These findings indicate the integrity of intestinal barrier (Figure 2). Interestingly, in ISO TX controls at 5 to 7 days posttransplant, low, but detectable, levels of OVA were assessed, with a trend to show higher levels of OVA compared with ISO controls at later time points. These results could be due to some permeability differences accounting for incomplete barrier reparation upon transplant operation or to denervation performed for transplantation or both. On the other hand, even at 5 POD, when only mild histopathological changes were detected, enteral administered OVA reach the circulation, resulting in levels higher than $1 \mu \mathrm{g} / \mathrm{mL}$, indicating leakage of the intestinal barrier caused by ACR. The seminal work of Grant et $\mathrm{al}^{5}$ have shown increase in graft permeability in HITx model using the administration of ethylenediaminetetraacetic acid $51 \mathrm{Cr}$ by enteral route and urinary excretion as measurement of barrier dysfunction. Although the tracer used by Grant et al is different than the tracer that we used, results are coincident, indicating the loss of barrier function during ACR process. In our study, the kinetics indicates that this is an early feature of ACR. The loss of barrier function and the putative passage of microbiotaderived biomolecules have been proposed as an amplification mechanism that contributes to increase inflammation along ACR process. ${ }^{30}$ Our results indicate that this mechanism is operating since the early stages of ACR process, which may provide the rationale for early therapeutic interventions in the clinics. In the clinical setting, our group showed that albumin levels in plasma can be used as a tool to assess mucosal barrier recovery after severe exfoliative rejection. ${ }^{39}$

From the beginning of ITx, it has been observed in the clinical practice that the development of ACR is associated with a progressive reduction of the intestinal motility to the point that significant ileus is observed in moderate and severe rejection, and the functional motility recovery is also a clinical indicator of recovery. ${ }^{33}$ This observation has been also described in animal models, finding a correlation with the severity of rejection and the participation of muscle layer myeloid cells in the physiopathology of the alteration. ${ }^{40}$ We also observed a correlation between ACR progression and loss of contractile capacity (Figure 2). Although our model has several limitations compared with the clinical ITx process of rejection itself, and currently lacks immunosuppressive therapy, it is an adequate model to start building knowledge. As it was mentioned, the main aim of the work was achieved: a rejection ITx rat model that further allows studying dynamic phenomena related with ACR at the different layers of the intestinal graft was established. We further observed an early rise in plasma nitrites associated to ACR progression (Figure 7), which was also shown in the clinical setting; and a late rise in plasma protein carbonyls that are produced during acute inflammatory processes that takes place in advanced stages of rejection (Figure 6). ${ }^{41}$

\section{Concluding Remarks}

In summary, we observed that functional features of the graft, such as glucose and OVA absorption, are altered at early stages of ACR, suggesting that they could be interesting parameters to evaluate in the clinical setting to provide additional tools for rejection diagnosis. The model provides a consistent kinetic that may allow to study not only the immunobiology of the ACR process but also to further study 
intervention strategies and search for biomarkers that could be translated to the clinical setting.

\section{ACKNOWLEDGMENTS}

The authors thank M.C Pallanza from INIBIOLP for the technical assistance in the measurement of nitrites and protein carbonyls; C. Ledesma from LTO for the maintenance and care of animals and GN Contigiani from Roche Diabetes Care-Argentina by support for absorption study.

\section{REFERENCES}

1. von Websky MW, Kitamura K, Ludwig-Portugall I, et al. Recombinant HLA-G as tolerogenic immunomodulant in experimental small bowel transplantation. PLoS One. 2016;11:e0158907.

2. Pech T, von Websky M, Ohsawa I, et al. Intestinal regeneration, residual function and immunological priming following rescue therapy after rat small bowel transplantation. Am J Transplant. 2012;12(Suppl 4):S9-S17.

3. Andres AM, Santamaria M, Hernandez-Oliveros F, et al. Difficulties, guidelines and review of developing an acute rejection model after rat intestinal transplantation. Transpl Immunol. 2016;36:32-41.

4. Nakao A, Tahara K, Inoue S, et al. Experimental models of small intestinal transplantation in rats: orthotopic versus heterotopic model. Acta Med Okayama. 2002;56:69-74.

5. Grant D, Zhong R, Hurlbut D, et al. A comparison of heterotopic and orthotopic intestinal transplantation in rats. Transplantation. 1991;51:948-954.

6. Yang Y, Song HL, Zhang W, et al. Heme oxygenase-1-transduced bone marrow mesenchymal stem cells in reducing acute rejection and improving small bowel transplantation outcomes in rats. Stem Cell Res Ther. 2016;7:164.

7. Okamoto T, Okamoto S, Fujimoto Y, et al. Suppression of acute rejection by administration of prostaglandin E2 receptor subtype 4 agonist in rat organ transplantation models. J Surg Res. 2013;183:852-859.

8. Xu H, Firdawes S, Yamamoto A, et al. Effects of blocking the chemokine receptors, CCR5 and CXCR3, with TAK-779 in a rat small intestinal transplantation model. Transplantation. 2008;86:1810-1817.

9. Yang JJ, Feng F, Hong L, et al. Interleukin-17 plays a critical role in the acute rejection of intestinal transplantation. World J Gastroenterol. 2013;19:682-691.

10. Guo WH, Tian L, Chan KL, et al. Role of CD4+ and CD8+ T cells in early and late acute rejection of small bowel allograft. J Pediatr Surg. 2001; 36:352-356.

11. Rumbo M, Chirdo FG, Fossati CA, et al. Analysis of structural properties and immunochemical reactivity of heat-treated ovalbumin. J Agric Food Chem. 1996;44:3793-3798.

12. Wu T, Abu-Elmagd K, Bond $\mathrm{G}$, et al. A schema for histologic grading of small intestine allograft acute rejection. Transplantation. 2003;75:1241-1248.

13. He G, Hart J, Thistlethwaite JR, et al. Inhibition of intestinal allograft rejection by an anti-CD 8 monoclonal antibody is not mediated by depletion alone. Transplant Proc. 1998;30:1069-1070.

14. Park PO, Haglund U, Bulkley GB, et al. The sequence of development of intestinal tissue injury after strangulation ischemia and reperfusion. Surgery. 1990;107:574-580.

15. Montes GS. Structural biology of the fibres of the collagenous and elastic systems. Cell Biol Int. 1996;20:15-27.

16. Rumbo M, Sierro F, Debard N, et al. Lymphotoxin beta receptor signaling induces the chemokine CCL20 in intestinal epithelium. Gastroenterology. 2004; 127:213-223.

17. Anderle P, Rumbo M, Sierro F, et al. Novel markers of the human follicleassociated epithelium identified by genomic profiling and microdissection. Gastroenterology. 2005;129:321-327.

18. Miranda KM, Espey MG, Wink DA. A rapid, simple spectrophotometric method for simultaneous detection of nitrate and nitrite. Nitric Oxide. $2001 ; 5: 62-71$.
19. Reznick AZ, Cross CE, Hu ML, et al. Modification of plasma proteins by cigarette smoke as measured by protein carbonyl formation. Biochem J. 1992;286:607-611.

20. Piao D-X, Jiang T, Liu L-X, et al. Formation of microchimerism in rat small bowel transplantation by splenocyte infusion. World J Gastroenterol. 2006;12:4166-4169.

21. Frezza EE, Gerunda GE, Fassina A, et al. NK activity during graft-versushost disease and graft rejection in rats following intestinal semiallogenic and allogenic transplantation with or without mesenteric lymphadenectomy. Transplantation. 1994;58:698-701.

22. Xu X, Gao X, Zhao X, et al. PU.1-silenced dendritic cells induce mixed chimerism and alleviate intestinal transplant rejection in rats via a Th1 to Th2 shift. Cell Physiol Biochem. 2016;38:220-228.

23. Yang $\mathrm{Y}$, Song $\mathrm{HL}$, Zhang $\mathrm{W}$, et al. Reduction of acute rejection by bone marrow mesenchymal stem cells during rat small bowel transplantation. PLoS One. 2014;9:e114528.

24. Lauro A, Marino IR, Matsumoto CS. Advances in allograft monitoring after intestinal transplantation. Curr Opin Organ Transplant. 2016;21: 165-170.

25. Kaufman SS, Lyden ER, Marks WH, et al. Lack of utility of intestinal fatty acid binding protein levels in predicting intestinal allograft rejection. Transplantation. 2001;71:1058-1060.

26. Pironi L, Guidetti M, Lauro A, et al. Plasma citrulline after small bowel transplantation: effect of time from transplantation, acute cellular rejection, and renal failure. Clin Transplant. 2015;29:1039.

27. Schaefer N, Tahara K, Pech T, et al. Inducible nitric oxide synthase expression in the intestinal muscularis mediates severe smooth muscle dysfunction during acute rejection in allogenic rodent small bowel transplantation. J Surg Res. 2008;150:159-168.

28. Talayero P, Mancebo E, Calvo-Pulido J, et al. Innate lymphoid cells groups 1 and 3 in the epithelial compartment of functional human intestinal allografts. Am J Transplant. 2016;16:72-82.

29. Pech T, Finger T, Fujishiro J, et al. Perioperative infliximab application ameliorates acute rejection associated inflammation after intestinal transplantation. Am J Transplant. 2010;10:2431-2441.

30. He G, Hart J, Thistlethwaite JR, et al. Modified surgical model of paratopic small bowel transplantation in mice. J Surg Res. 1998;80:188-193.

31. Fishbein TM. Intestinal transplantation. N Engl J Med. 2009;361: 998-1008.

32. Loo L, Vrakas G, Reddy S, et al. Intestinal transplantation: a review. Curr Opin Gastroenterol. 2017;33:203-211.

33. Boluda ER. Pediatric small bowel transplantation. Curr Opin Organ Transplant. 2015;20:550-556.

34. Trentadue G, Dijkstra G. Current understanding of alloimmunity of the intestinal graft. Curr Opin Organ Transplant. 2015;20:286-294.

35. Chang C-H, Curtis JD, Maggi LB, et al. Posttranscriptional control of Tcell effector function by aerobic glycolysis. Cell. 2013;153:1239-1251.

36. Simionescu M, Gafencu A, Antohe F. Transcytosis of plasma macromolecules in endothelial cells: a cell biological survey. Microsc Res Tech. 2002; 57:269-288.

37. Weiner ML. Intestinal transport of some macromolecules in food. Food Chem Toxicol. 1988;26:867-880.

38. Heyman M. Gut barrier dysfunction in food allergy. Eur J Gastroenterol Hepatol. 2005;17:1279-1285.

39. Zambernardi A, Gondolesi G, Cabanne A, et al. Serum albumin level during intestinal exfoliative rejection: a potential predictor of graft recovery and patient outcome. Clin Transplant. 2013;27:E137-E142.

40. Schaefer N, Tahara K, von Websky M, et al. Role of resident macrophages in the immunologic response and smooth muscle dysfunction during acute allograft rejection after intestinal transplantation. Transpl Int. 2008;21: 778-791.

41. Yamayoshi $Y$, Watanabe T, Tanabe M, et al. Novel application of ProteinChip technology exploring acute rejection markers of rat small bowel transplantation. Transplantation. 2006;82:320-326. 